\title{
An Experimental Model of Breast Cancer Cells: Informative Protocol for In Vitro Culture
}

\author{
XINXIN DU ${ }^{1}$, KRISTINA KLASCHIK $^{2,3}$, PETER MALLMANN ${ }^{1}$, EVGENIA ISACHENKO ${ }^{1}$, \\ GOHAR RAHIMI $^{1}$, YUE ZHAO ${ }^{4}$, CHRISTIANE BRUNS ${ }^{4}$ and VLADIMIR ISACHENKO ${ }^{1}$ \\ ${ }^{1}$ Research Group for Reproductive Medicine, IVF-Laboratory and Department of Gynecology, \\ University of Cologne, Cologne, Germany; \\ ${ }^{2}$ Center for Familial Breast and Ovarian Cancer, University Hospital Cologne, Cologne, Germany; \\ ${ }^{3}$ Center for Integrated Oncology, University Hospital Cologne, Medical Faculty, Cologne, Germany; \\ ${ }^{4}$ Department of General, Visceral and Cancer Surgery, University of Cologne, Cologne, Germany
}

\begin{abstract}
Background: Cryopreservation of ovarian tissue for patients with cancer comes with the concern for the existence of cancer cells in the grafts. Data about cancer cell viability after cryopreservation are currently lacking. For the experimental study of cryo-stability of cancer cells, different protocols of in vitro culture of various cell lines are used. The existing protocols are not able to mimic the effectiveness of in vivo/in situ development of cancer cells. This study aimed to test the new protocol for in vitro culture of breast cancer cells. Materials and Methods: ZR-75-1 and MDA-MB-231 cells were in vitro-cultured in standard [RPMI 1640 and Dulbecco's modified Eagle's medium (DMEM), respectively] and experimental (AIM V) medium. Cell phenotypes were tested by CCK-8 cell proliferation measurement, wound-healing assay, transmembrane cell migration and invasion assay and immunofluorescent staining. A 10-day in vitro culture without cell passaging was conducted on both cell lines in culture medium to observe the generation of a cell layer as a model of solid tumor tissue. The density of the cell layers was revealed by immunofluorescent staining. Results: AIM V significantly promoted continued proliferation of both cell lines. Cell motility of ZR-75-1 cells was increased considerably more in AIM $V$ than in RPMI-1640. For MDA-MB-231 cells cultured in AIM $V$ and DMEM, no significant differences in mobility and invasion were observed. Both cell lines maintained in AIM V medium generated a cell layer on day 7 and formed a compact structure on day 10 of in vitro culture. Conclusion: In vitro culture of ZR-75-1 and
\end{abstract}

Correspondence to: Vladimir Isachenko, Kerpener Str. 34, 50931 Cologne, Germany. Tel: +49 2214784924, Fax: +49 2214782656, e-mail:v.isachenko@yahoo.com

Key Words: Breast neoplasm, experimental model, in vitro techniques, cell proliferation, cell motility, neoplasm invasiveness.
MDA-MB-231 cells in AIM V medium is more informative than culture in standard medium. The described protocol provides a means for the formation of compact structures from in vitro-cultured cancer cells as a model of solid tumor tissue.

Along with the improvement of anticancer treatments, the 5-year survival rate of patients has increased over the past decades (1). Cryopreservation of ovarian tissue (freezing and thawing) and transplantation have become an established technique with promising results for preserving fertility in cancer survivors $(2,3)$. However, clinicians are concerned about the presence of neoplastic cells in the preserved tissues because these tissues were removed before anticancer treatment. After thawing and transplantation, neoplastic cells might be re-introduced to a cancer-free body. The risk is high not only for leukemic patients (4-6); cancer relapse was reported after transplantation of cryopreserved ovarian tissue in a patient with breast cancer (7).

A few studies mainly performed histological analysis combined with immunohistochemistry and reverse transcription polymerase chain reaction to detect the existence of metastatic breast cancer cells in ovarian tissue at three time points: (i) biopsy before freezing $(8,9)$; (ii) immediately after thawing $(10,11)$; (iii) after xenotransplantation following thawing $(12,13)$. The major controversy is that no evidence of primary disease recurrence was found in severe combined immunodeficient mice carrying ovarian fragments from patients with breast cancer although particular biomarkers were expressed $(11,13)$. Consequently, clinicians might overestimate the risk of cancer cell reintroduction and subsequent transplantation will be contraindicated for some patients (14). Cryopreservation affects the functional state of particular types of cells and results in their apoptosis (15-17). However, data about cancer cell viability after cryopreservation are currently lacking. For experimental study of cryo-stability of cancer cells, different protocols for in vitro 


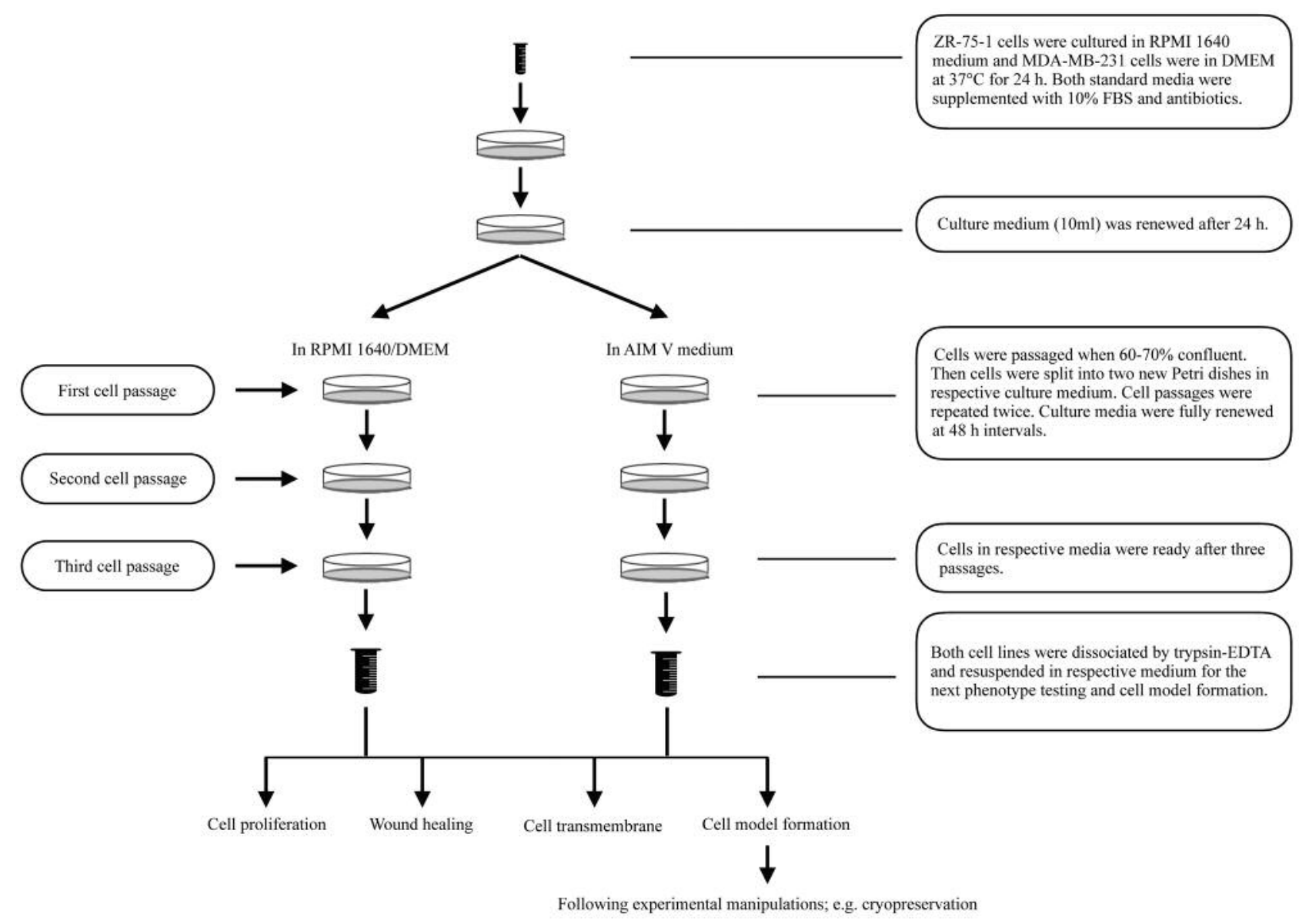

Figure 1. Design of experiments.

culture of various cell lines are used. The existing protocols are not able to mimic the effectiveness of in vivo/in situ development of cancer cells.

In this study, we aimed to test a new protocol for in vitro culture of breast cancer cells.

\section{Materials and Methods}

Cell lines and culture conditions. Except where otherwise specified, all reagents were obtained from Sigma Chemical Co. (St. Louis, MO, USA).

The study design is presented in Figure 1. ZR-75-1 and MDAMB-231 cell lines were purchased from American Type Culture Collection (Manassas, Virginia, USA) and grown in RPMI 1640 medium with GlutaMAX ${ }^{\mathrm{TM}}$ (Thermo Fisher Scientific, Waltham, MA, USA) and Dulbecco's modified Eagle's medium (DMEM) supplemented with $10 \%$ fetal bovine serum (FBS) (Biochrom $\mathrm{GmbH}$, Berlin, Germany) and antibiotics at $37^{\circ} \mathrm{C}$ in a humidified chamber with $5 \% \mathrm{CO}_{2}$, respectively. For all experiments, unless otherwise specified, AIM V medium (Thermo Fisher Scientific) supplemented with $10 \%$ FBS and amphotericin B was used for both cell lines after the culture became $60-70 \%$ confluent.
Cell proliferation measurement. ZR-75-1 and MDA-MB-231 cells were seeded both in the control medium and AIM V at a density of $1 \times 10^{4}$ cells $/ \mathrm{ml}$ in 96 -well plates. Cells were allowed to adhere overnight. Cell proliferation was measured by Cell Counting Kit- 8 (CCK-8) solution consecutively for 7 days. From day 1 to $7,10 \mu \mathrm{l}$ CCK-8 solution was added to each well of one plate at a fixed time and incubated for $4 \mathrm{~h}$, then the OD at $450 \mathrm{~nm}$ (reference $650 \mathrm{~nm}$ ) was determined by a multimode reader machine (Tecan Group Ltd., Maennedorf, Zurich, Switzerland). Culture media were renewed every $48 \mathrm{~h}$. Results were plotted with the time axis as the abscissa and the cell count as the vertical axis. Each experiment was repeated three times.

Wound-healing assay. Wound-healing assay was carried out to determine the cell mobility and migration of tumor cells. ZR-75-1 and MDA-MB-231 cell suspensions in initial medium and AIM V were adjusted to a density of $1 \times 10^{6} \mathrm{cells} / \mathrm{ml}$ and $70 \mu \mathrm{l}$ was seeded into each well of a $35 \mathrm{~mm} \mu$-Dish ibidi Culture Insert (ibidi GmbH, Planegg, Bavaria, Germany) then incubated for $24 \mathrm{~h}$ to obtain a confluent cell layer. After removal of the insert, cells were washed with PBS twice to remove cell debris and non-attached cells. Then the $\mu$-Dish was filled with $2 \mathrm{ml}$ of $1 \%$ FBS-supplemented cell-free medium. Timelapse measurement of the wound area of ZR-75-1 and MDA-MB-231 
cell layer was performed every $24 \mathrm{~h}$ and every $2 \mathrm{~h}$, respectively, in order to calculate cell front velocity until complete confluence. Experiments were carried out in triplicate at least three times.

Transmembrane cell migration and invasion. Cell invasion was determined in vitro to evaluate the ability of tumor cells to transmigrate a layer of reconstructed extracellular matrix. Corning transwell inserts were used to carry out the cell invasion assay. Polycarbonate filters $(6.5 \mathrm{~mm}$ in diameter, $8 \mu \mathrm{m}$ pore size $)$ were coated with type I rat tail collagen $(100 \mu \mathrm{g} / \mathrm{ml}$; BD Biosciences, Franklin Lakes, NJ, USA) for $1 \mathrm{~h}$ at $37^{\circ} \mathrm{C}$ in accordance with the manufacturer's protocol. ZR-75-1 and MDA-MB-231 cells were resuspended and seeded into the upper compartment of the insert in serum-free culture medium. The lower chambers were filled with $600 \mu \mathrm{l}$ of the appropriate culture medium supplemented with FBS as a chemoattractant. ZR-75- 1 cells were seeded at $2 \times 10^{5}$ cells/well for $72 \mathrm{~h}$ at $37^{\circ} \mathrm{C}$ in a $5 \% \mathrm{CO}_{2}$ incubator using $20 \% \mathrm{FBS}$ as a chemoattractant; MDA-MB-231 cells were seeded $5 \times 10^{4}$ cells/well for $8 \mathrm{~h}$ using $10 \% \mathrm{FBS}$ as a chemoattractant. After incubation, the upper inserts with cells were washed with PBS and fixed with $4 \%$ formaldehyde and permeabilized with methanol at room temperature. Cells were then stained with $0.1 \%$ crystal violet solution and were gently rinsed with PBS and wiped by cottontipped swabs then dried in the air. Invading cells went through the polymerized collagen layer to the bottom of the polycarbonate membranes and were counted in five different fields of view under a microscope. For the migration assay, cells were treated using the same procedure, except that the transwell membrane was not coated with collagen. Samples in each group ran in triplicate. Each experiment was performed at least three times.

Model formation. After three cell passages, ZR-75-1 and MDA-MB231 cells maintained in respective medium were in vitro-cultured for 10 days without passaging. The culture medium was renewed every $24 \mathrm{~h}$ after a cell layer was formed. A cell scraper (Greiner Bio-one, Frickenhausen, Baden-Wuerttemberg, Germany) was used for harvesting the cell layer as a model of solid tumor tissue for subsequent experimental manipulations, e.g. cryopreservation.

Immunofluorescence of the cell layers. Both cell lines were cultured in a 6-well plate with a cover glass. After a dense cell layer was formed on the cover glass, the cells were fixed with $2 \%$ paraformaldehyde in PBS for $15 \mathrm{~min}$ at room temperature. After two washes with PBS, the cells were permeabilized with $0.5 \%$ Triton X100 in PBS for 10 min. Cells were washed again twice with PBS and then blocked with Cell Staining Buffer (Biolegend, San Diego, CA, USA) for $30 \mathrm{~min}$. The glass covers were transferred into a humidified chamber and then incubated with Alexa Fluor 488conjugated Flash Phalloidin (Biolegend) in PBS (1:20) for $1 \mathrm{~h}$. The glass covers were then washed twice with PBS and further counterstained with 4',6-diamidino-2-phenylindole. The following day, the slides were visualized using a Leica SP8 confocal microscope and images were taken using LAS X software (Leica Microsystems, Wetzlar, Hesse, Germany).

Statistical analysis. Data analysis was executed with SPSS 23.0 software (IBM Corp., Armonk, NY, USA). Differences among the groups were tested by Student's $t$-test or two-way ANOVA. Data are expressed as mean \pm standard deviation (SD). The level of statistical significance was set at $p<0.05$.
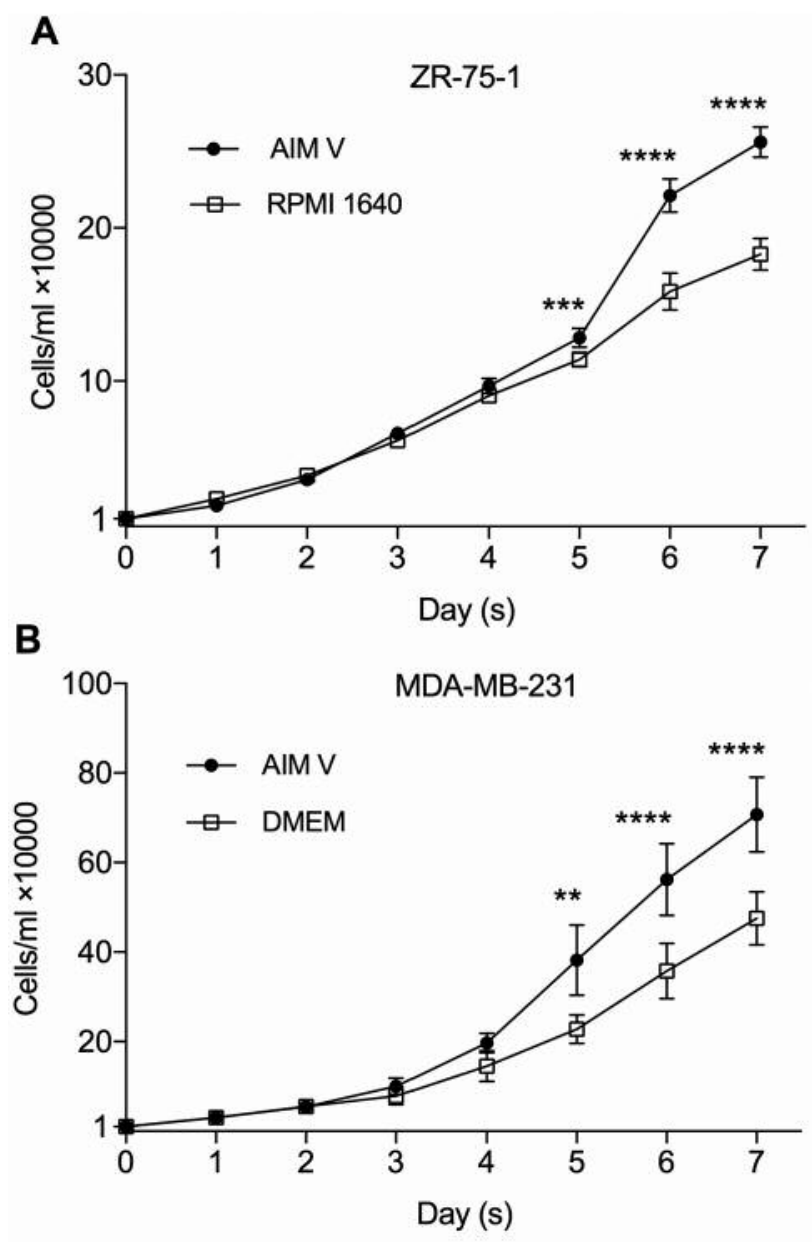

Figure 2. Cell viability of ZR-75-1 and MDA-MB-231 cells in standard culture medium and AIM V. Graphical representations of cell viability data showing significant promotion of proliferation of ZR-75-1 (A) and $M D A-M B-231$ cells $(B)$ when maintained in standard medium and AIM V. DMEM: Dulbecco's modified Eagle's medium. Significantly different at $* * p<0.01, * * * p<0.001$, and $* * * * p<0.0001$.

\section{Results}

AIM V medium promotes cell proliferation. Cells were seeded in seven 96-well culture plates at a consistent density and were adhered after incubating for $24 \mathrm{~h}$ at $37^{\circ} \mathrm{C}$ in a $5 \%$ $\mathrm{CO}_{2}$ incubator. From day 1 to day $7,10 \mu \mathrm{CCK}-8$ solution was added to each well of one plate at a fixed time and incubated for $4 \mathrm{~h}$, then the OD was determined. As shown in Figure 2, AIM V significantly promoted cell proliferation of ZR-75-1 and MDA-MB-231 cells in a time-dependent fashion. Cells maintained in AIM V proliferated markedly faster after 5 days culture and reached a high density after 7 -day in vitro culture $\left(2.56 \times 10^{5}\right.$ and $7.07 \times 10^{5}$ cells $/ \mathrm{ml}$, respectively), with significant differences compared to cells in the control media. 


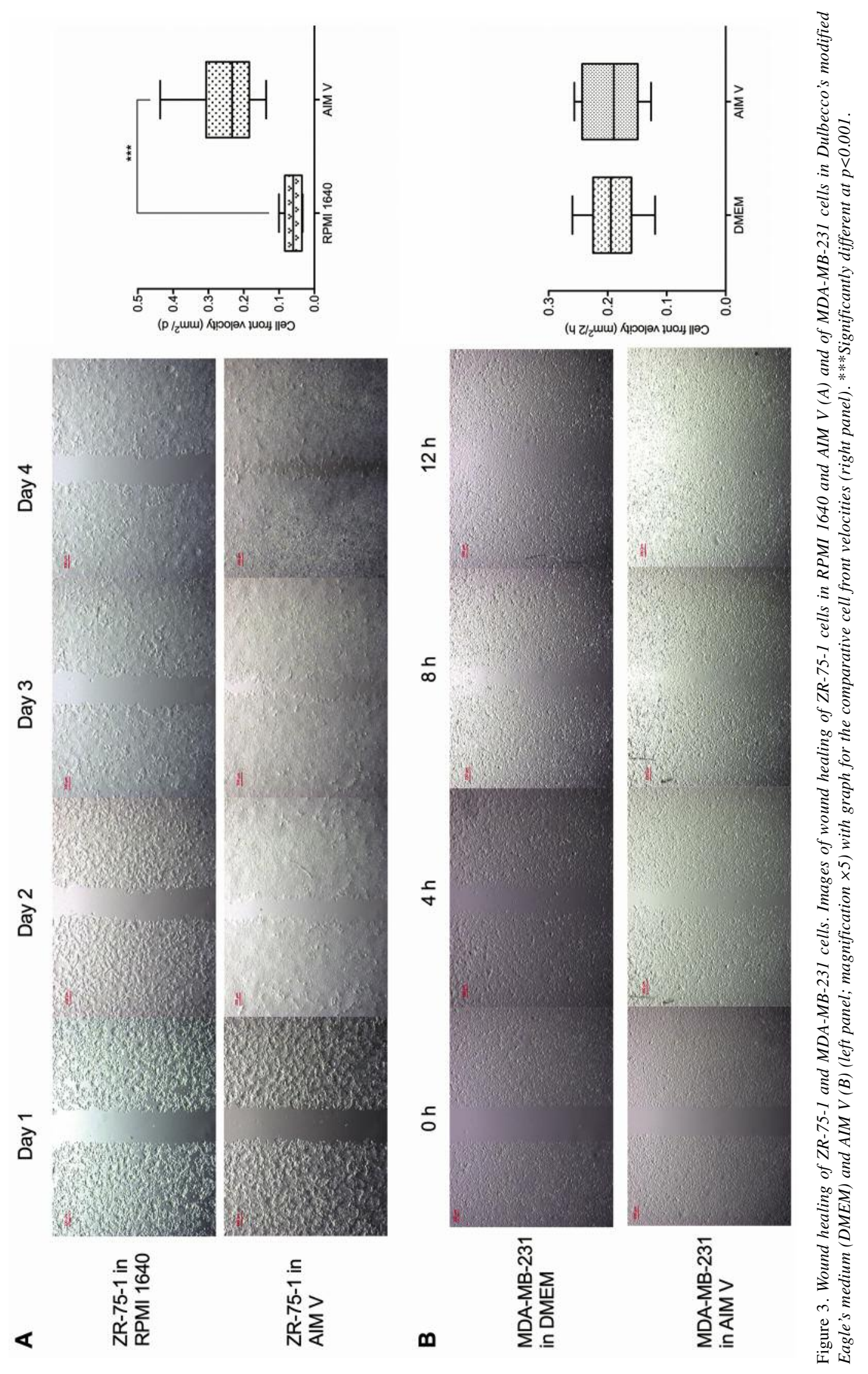



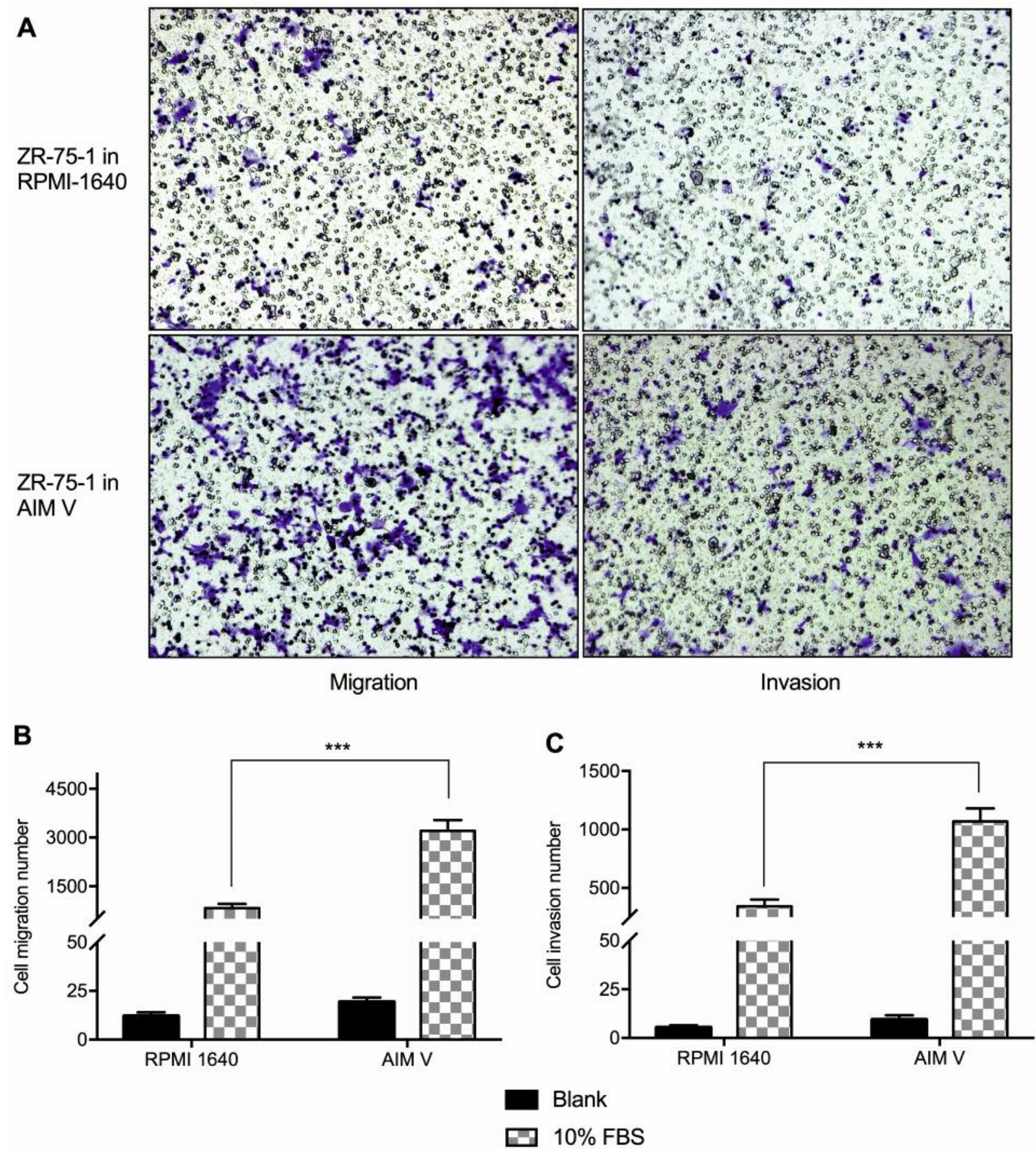

Figure 4. Transmembrane migration and invasion of ZR-75-1 cells. A: Images of transmembrane migration and invasion of ZR-75-1 cells in RPMI 1640 and AIM V (magnification, $\times 13.5)$. B: Graphical representation of transmembrane migration of ZR-75-1 cells in AIM V compared with cells in RPMI 1640. C: Graphical representation of transmembrane invasion of ZR-75-1 cells in AIM V compared with cells in RPMI 1640. ***Significantly different at $p<0.001$.

AIM V medium increases wound healing of ZR-75-1 cells. Active cell mobility is an essential step in tumor cell metastasis leading tumor cell migration to distant sites. Cell movement was measured using a well-established wound-healing assay. For ZR-75-1 and MDA-MB-231 cells, photographs were taken under a microscope at intervals until complete confluence. The data indicate that the wound area of ZR-75-1 cells in AIM V medium 


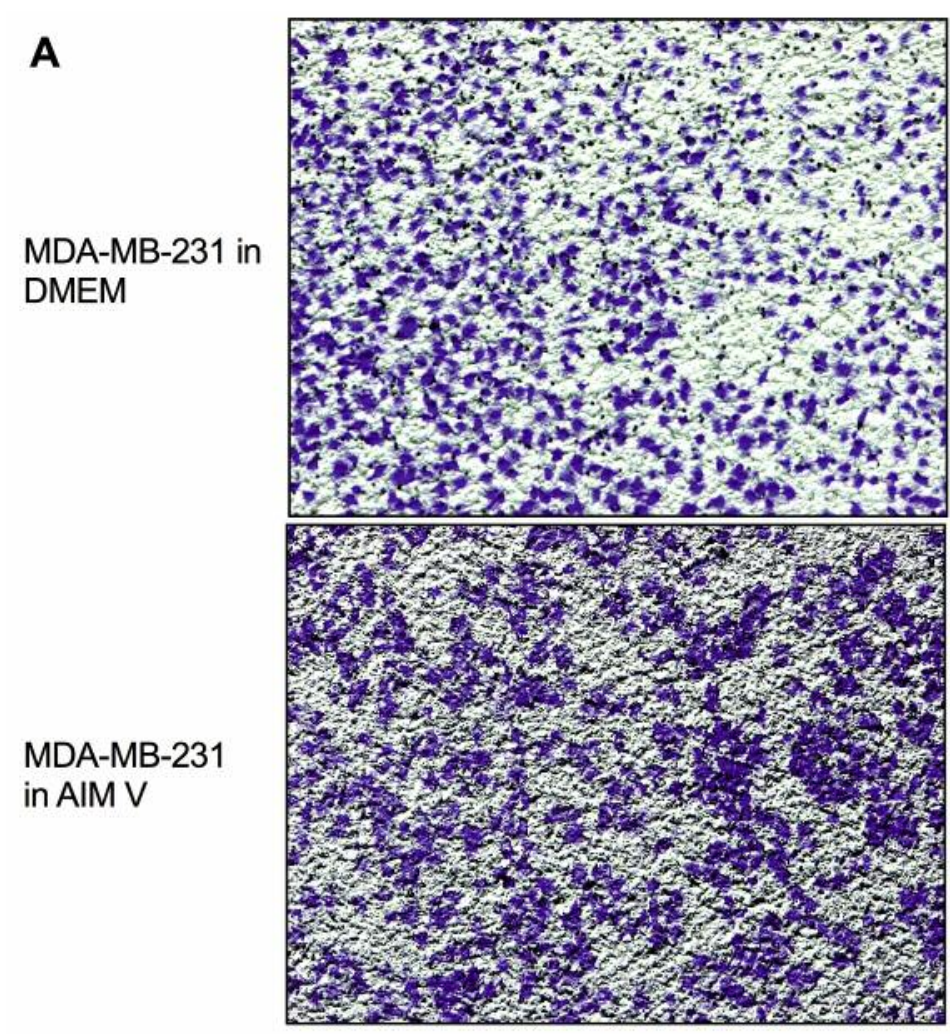

Migration

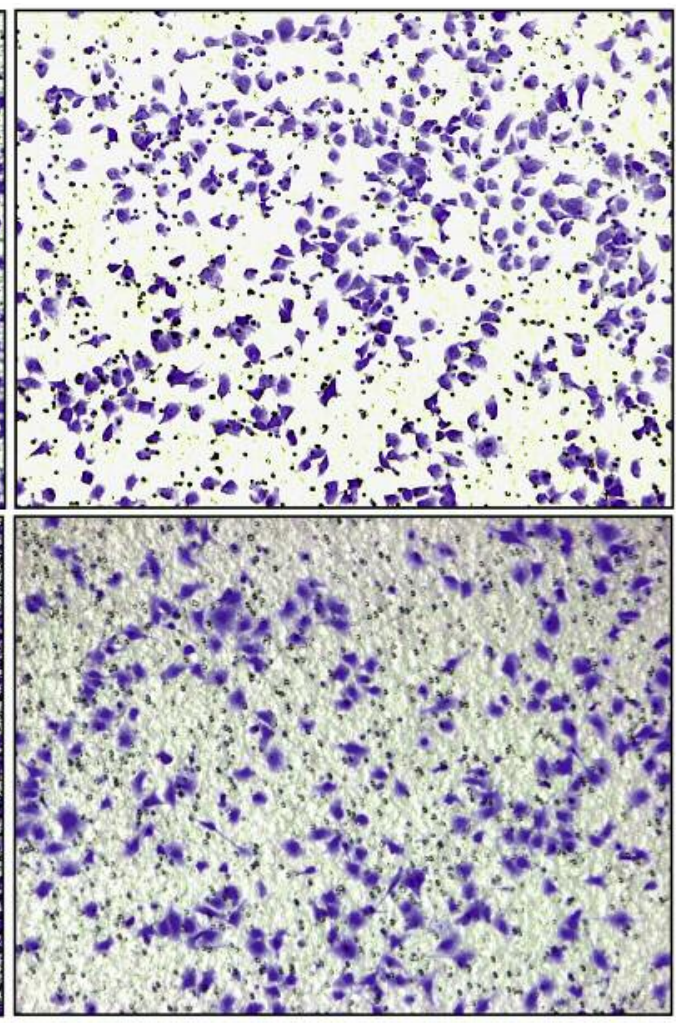

Invasion
B

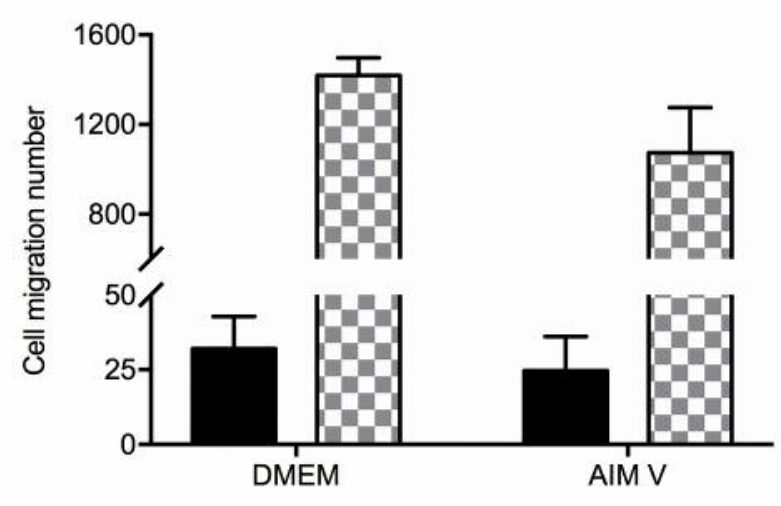

C

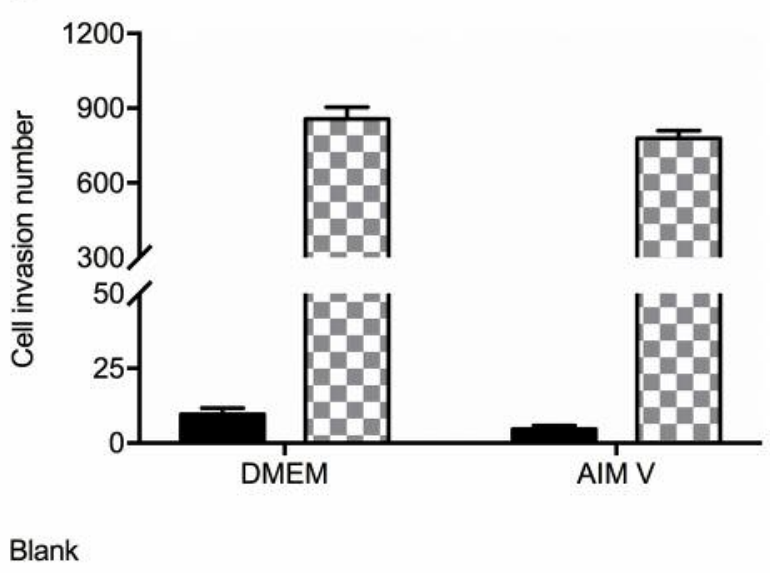

$10 \%$ FBS

Figure 5. Transmembrane migration and invasion of MDA-MB-231 cells. A: Images of transmembrane migration and invasion of MDA-MB-231 cells in Dulbecco's modified Eagle's medium (DMEM) and AIM V (magnification, x13.5). B: Graphical representation of transmembrane migration of MDA-MB-231 cells in AIM V compared to cells in DMEM. C: Graphical representation of transmembrane invasion of MDA-MB-231 cells in AIM V compared to cells in DMEM.

decreased much more than that in RPMI-1640 $(p<0.01)$. For MDA-MB-231 cells, no significant difference was observed between cell migration in DMEM and AIM V, as shown in Figure 3.
The medium affects migration and invasion of ZR-75-1 and $M D A-M B-231$ cells. Invasion of the basement membranes is another critical event in tumor metastasis. In order to study cell motility and the invasive ability of tumor cells without 


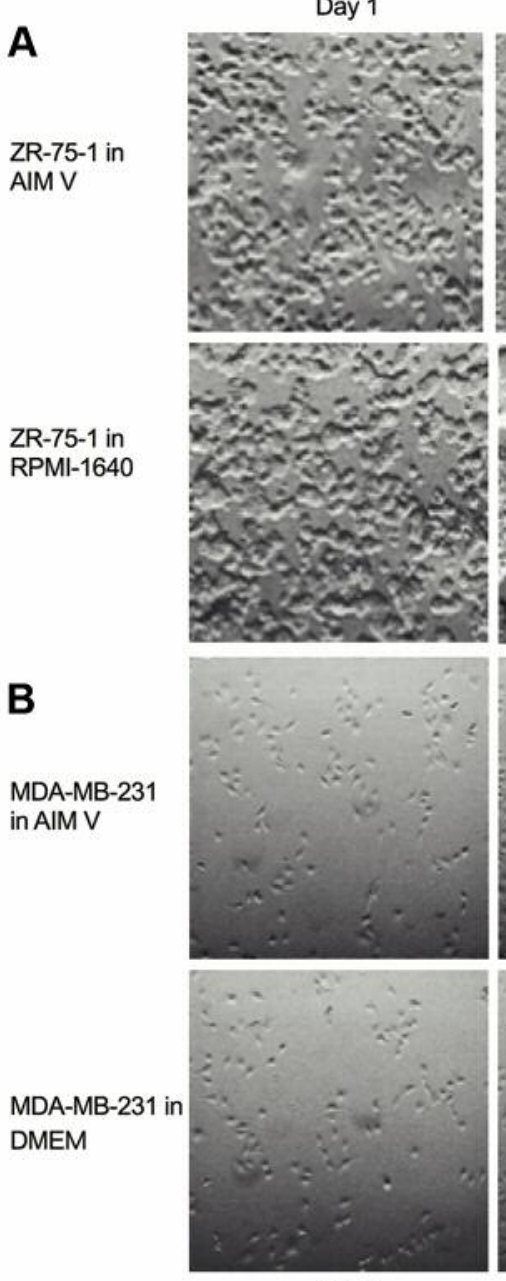

DAPI

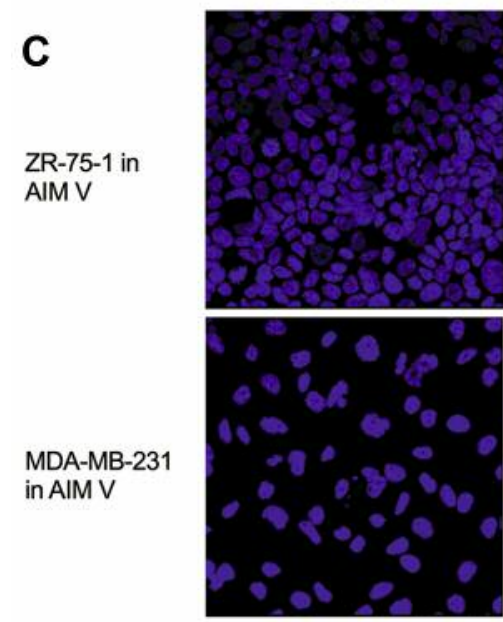

Day 4
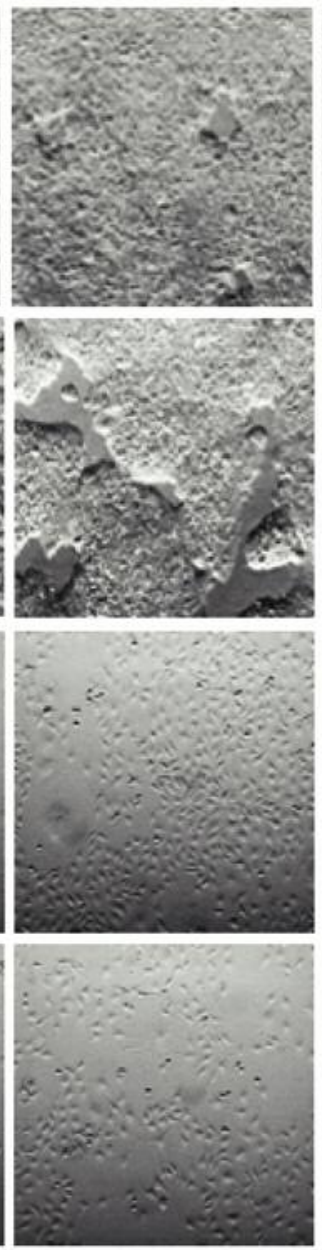

F-Actin

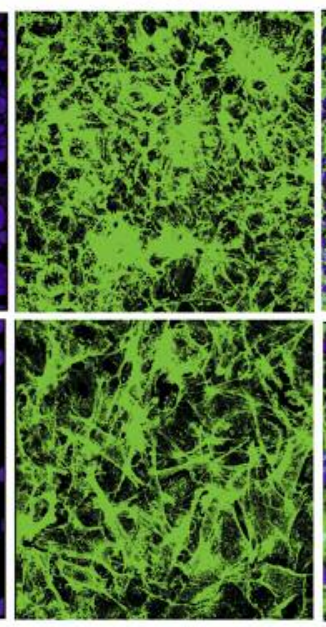

Day 7
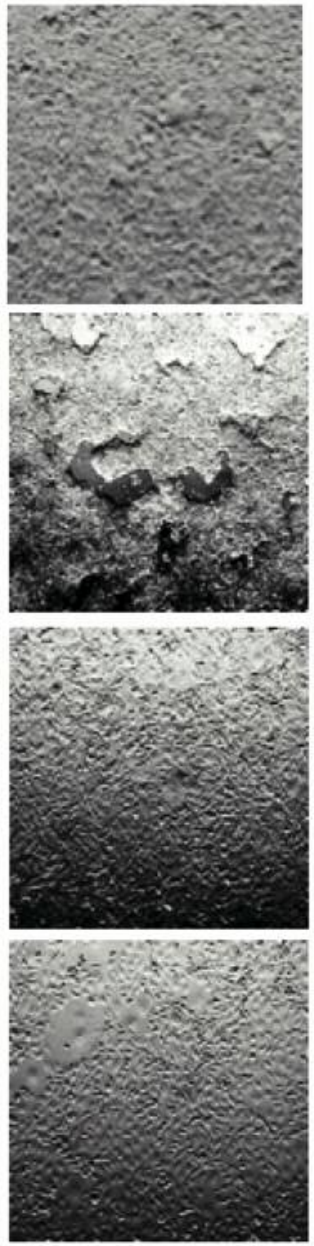

Merge
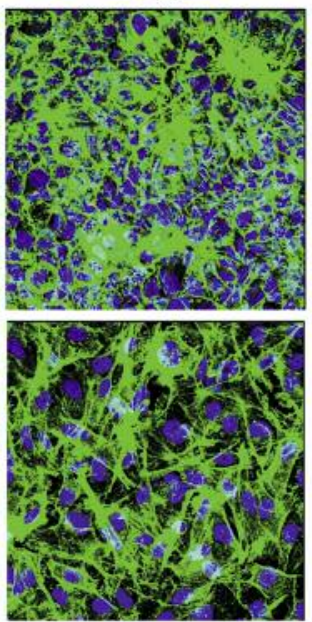

Day 10
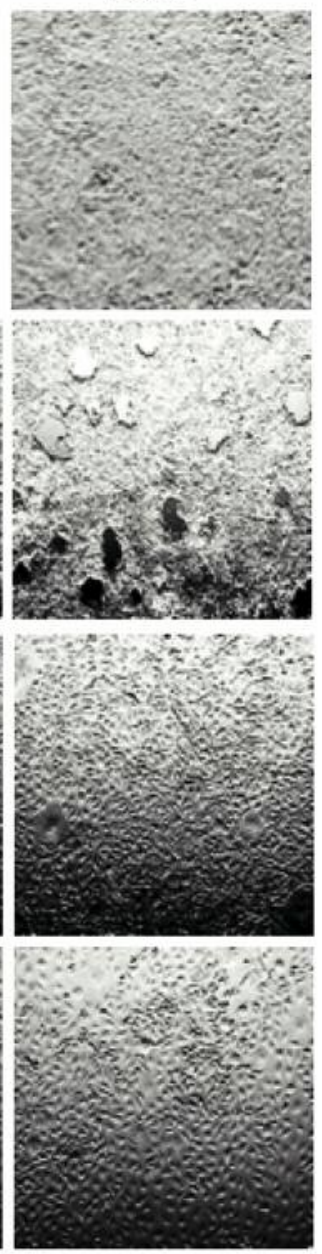

Cell model
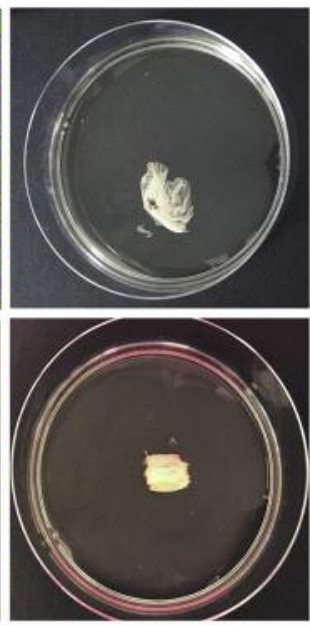

Figure 6. Formation of the compact structured cell models. A: Representative images of the formation of the ZR-75-1 cell layer in AIM $V$ supplemented with 10\% fetal bovine serum (FBS) (upper blocks). Cells in RPMI 1640 did not become $100 \%$ confluent during 10 days of in vitro culture (lower blocks). B: Representative images of the formation of the MDA-MB-231 cell layer during 10 days of in vitro culture in AIM V supplemented with 10\% FBS (upper blocks). We observed 100\% confluence of the cells in DMEM, albeit the cell layer was loose (lower blocks). C: Immunofluorescence in ZR-75-1 (upper blocks) and MDA-MB-231 (lower blocks) cell layers before harvest and the compact structured cell model in AIM V. No cell adhered in the culture dishes. DAPI: 4',6-Diamidino-2-phenylindole. 
the effect of serum-induced cell proliferation in different in vitro culture environments, we performed a transmembrane migration and invasion assay on both cell lines using FBS as a chemoattractant. Staining images of the invasive cells were obtained and presented as photographic evidence. Differences in cell migration and invasion were observed in this analysis. ZR-75-1 cells displayed a significantly increased transmembrane ability in AIM V compared with that in RPMI 1640 (Figure 4) $(p<0.001)$. For MDA-MB-231 cells, the transmembrane ability slightly decreased in AIM V compared with that in DMEM, without statistical significance $(p>0.05)$ (Figure 5).

Formation of the cell model of solid tumor tissues. After the assessment of cellular properties in different medium, we found that ZR-75-1 and MDA-MB-231 cells maintained in AIM V showed significantly increased proliferation and reached a high cell density without loss of cell motility and metastatic ability. We observed the formation of a cell layer of both cell lines maintained in AIM V medium on day 7 and the generation of a compact structure from the cancer cells on day 10 of in vitro culture. The immunofluorescence images revealed the cell density before harvesting (Figure 6). ZR-75-1 and MDA-MB231 in the primary standard medium did not form a dense cell layer after in vitro culture during the same period.

\section{Discussion}

Ductal carcinoma is the most common histological category of breast cancer and luminal $\mathrm{A}$ is the major molecular subtype (18-20). For our study, we used two representative cell lines derived from invasive ductal breast carcinoma. Differences can be characterized by the following peculiarities: ZR-75-1 cell line with luminal A molecular subtype has mass aggregate morphology, while MDA-MB231 cells with basal molecular subtype has stellate aggregate morphology $(21,22)$. To our knowledge, we are the first to have constructed an experimental model of solid tumor tissues from in vitro-cultured breast cancer cells as an alternative of tumor tissue from patients. Our model can be widely applied because we obtained similar results after research of both cell lines.

AIM $\mathrm{V}$ is intended for human ex vivo tissue and cell culture processing applications and allows for high-density culture with no difference in cell viability. It is widely used for T-cells and dendritic cells, which showed better proliferation and migration when compared with culture in RPMI 1640 (23-25). In our study, we presupposed that the culture medium might affect the phenotype of cancer cells. It was demonstrated that viability, migration and invasion of ZR-75-1 cells maintained in AIM V were significantly promoted compared to that in the initial medium. Although AIM V did not have a similar effect on MDA-MB-231 cells, their cell motility did not significantly decline. We obtained the cell model of both cell lines after confluence to $100 \%$ with compact structures. This is the crucial factor in the subsequent experimental manipulation, e.g. programmed slow freezing to study cryo-stability of cancer cells.

Peek et al. made a model system by injecting cancer cells into ovarian cortex fragment, which led to the formation of small metastases in vitro (26). This model might facilitate the development of detection of isolated tumor cells or micrometastases in ovarian tissue or elimination of cancer cells. However, it is still difficult to explain the inconsistency between micro-and macro-level inspections. Moreover, it is vital to have a clear understanding of the effect of cryopreservation on cancer cell properties before purging such cells. It is optimal to eliminate malignant cells while leaving normal ovarian tissue unaffected (27). Our model could be applied to evaluate the effect of cryopreservation on cancer cells and to assess the risk of primary disease recurrence. Relevant tumor target treatments might be performed before ovarian tissue transplantation and thereby minimize side-effects on ovarian tissue viability $(28,29)$.

Breast cancer is the most frequent indication for cryopreservation of ovarian tissue and transplantation (30). However, the risk of ovarian metastasis of breast cancer in patients aged $21-30$ years is $19.4 \%$ and reaches to $25.0 \%$ in the 31- to 39-year-old age group (31). Therefore, it is essential to assess the risk of primary disease recurrence when fertility preservation is considered for breast cancer survivors after treatment.

In summary, in vitro culture of ZR-75-1 and MDA-MB231 cells in AIM V medium is more informative than their culture in standard medium. The described protocol provides a means for the formation of compact structures from in vitro-cultured cancer cells as a model of solid tumor tissues.

\section{References}

1 Keegan TH, Ries LA, Barr RD, Geiger AM, Dahlke DV, Pollock $\mathrm{BH}$ and Bleyer A; National Cancer Institute Next Steps for Adolescent and Young Adult Oncology Epidemiology Working Group: Comparison of cancer survival trends in the United States of adolescents and young adults with those in children and older adults. Cancer 122(7): 1009-1016, 2016.

2 Donnez J, Dolmans MM, Pellicer A, Diaz-Garcia C, Sanchez Serrano M, Schmidt KT, Ernst E, Luyckx V and Andersen CY: Restoration of ovarian activity and pregnancy after transplantation of cryopreserved ovarian tissue: A review of 60 cases of reimplantation. Fertil Steril 99: 1503-1513, 2013.

3 Detti L, Martin DC and Williams LJ: Applicability of adult techniques for ovarian preservation to childhood cancer patients. J Assist Reprod Genet 29: 985-995, 2012.

4 Rosendahl M, Andersen MT, Ralfkiaer E, Kjeldsen L, Andersen MK and Andersen CY: Evidence of residual disease in cryopreserved ovarian cortex from female patients with leukemia. Fertil Steril 94: 2186-2190, 2010. 
5 Dolmans MM, Marinescu C, Saussoy P, Van Langendonckt A Amorim $\mathrm{C}$ and Donnez J: Reimplantation of cryopreserved ovarian tissue from patients with acute lymphoblastic leukemia is potentially unsafe. Blood 116: 2908-2914, 2010.

6 Greve T, Clasen-Linde E, Andersen MT, Andersen MK, Sorensen SD, Rosendahl M, Ralfkiaer E and Andersen CY: Cryopreserved ovarian cortex from patients with leukemia in complete remission contains no apparent viable malignant cells. Blood 120: 4311-4316, 2012.

7 Ernst EH, Offersen BV, Andersen CY and Ernst E: Legal termination of a pregnancy resulting from transplanted cryopreserved ovarian tissue due to cancer recurrence. J Assist Reprod Genet 30: 975-978, 2013.

8 Azem F, Hasson J, Ben-Yosef D, Kossoy N, Cohen T, Almog B, Amit A, Lessing JB and Lifschitz-Mercer B: Histologic evaluation of fresh human ovarian tissue before cryopreservation. Int J Gynecol Pathol 29: 19-23, 2010.

9 Fabbri R, Vicenti R, Magnani V, Pasquinelli G, Macciocca M, Parazza I, Paradisi R, Battaglia C and Venturoli S: Cryopreservation of ovarian tissue in breast cancer patients: 10 years of experience. Future Oncol 8: 1613-1619, 2012.

10 Rosendahl M, Timmermans Wielenga V, Nedergaard L, Kristensen SG, Ernst E, Rasmussen PE, Anderson M, Schmidt KT and Andersen CY: Cryopreservation of ovarian tissue for fertility preservation: No evidence of malignant cell contamination in ovarian tissue from patients with breast cancer. Fertil Steril 95: 2158-2161, 2011.

11 Luyckx V, Durant JF, Camboni A, Gilliaux S, Amorim CA, Van Langendonckt A, Irenge LM, Gala JL, Donnez J and Dolmans MM: Is transplantation of cryopreserved ovarian tissue from patients with advanced-stage breast cancer safe? A pilot study. J Assist Reprod Genet 30: 1289-1299, 2013.

12 Rodriguez-Iglesias B, Novella-Maestre E, Herraiz S, DiazGarcia C, Pellicer N and Pellicer A: New methods to improve the safety assessment of cryopreserved ovarian tissue for fertility preservation in breast cancer patients. Fertil Steril 104: 14931502.e1491-1492, 2015.

13 Bockstaele L, Boulenouar S, Van Den Steen G, Dechene J, Tsepelidis S, Craciun L, Noel JC and Demeestere I: Evaluation of quantitative polymerase chain reaction markers for the detection of breast cancer cells in ovarian tissue stored for fertility preservation. Fertil Steril 104: 410-417.e414, 2015.

14 Courbiere B, Prebet T, Mozziconacci MJ, Metzler-Guillemain C, Saias-Magnan $\mathbf{J}$ and Gamerre M: Tumor cell contamination in ovarian tissue cryopreserved before gonadotoxic treatment: should we systematically exclude ovarian autograft in a cancer survivor? Bone Marrow Transplant 45: 1247-1248, 2010.

15 Hengstler JG, Utesch D, Steinberg P, Platt K, Diener B, Ringel M, Swales N, Fischer T, Biefang K and Gerl M: Cryopreserved primary hepatocytes as a constantly available in vitro model for the evaluation of human and animal drug metabolism and enzyme induction. Drug Metab Rev 32: 81-118, 2000.

16 Grondin M, Hamel F, Sarhan F and Averill-Bates DA: Metabolic activity of cytochrome p450 isoforms in hepatocytes cryopreserved with wheat protein extract. Drug Metab Dispos 36: 2121-2129, 2008

17 Cai G, Lai B, Hong H, Lin P, Chen W, Zhou Z and Chen H: Effects of cryopreservation on excretory function, cellular adhesion molecules and vessel lumen formation in human umbilical vein endothelial cells. Mol Med Rep 16: 547-552, 2017.
18 Dai X, Li T, Bai Z, Yang Y, Liu X, Zhan J and Shi B: Breast cancer intrinsic subtype classification, clinical use and future trends. Am J Cancer Res 5: 2929-2943, 2015.

19 Oloomi M, Moazzezy N and Bouzari S: Modulation of molecular biomarker expression in response to chemotherapy in invasive ductal carcinoma. Biomed Res Int 2018: 7154708, 2018.

20 Zheng L, Zhou B, Meng X, Zhu W, Zuo A, Wang X, Jiang R and $\mathrm{Yu} S$ : A model of spontaneous mouse mammary tumor for human estrogen receptor- and progesterone receptor-negative breast cancer. Int J Oncol 45: 2241-2249, 2014.

21 Ziperstein MJ, Guzman A and Kaufman LJ: Breast cancer cell line aggregate morphology does not predict invasive capacity. PLoS ONE 10: $\mathrm{e} 0139523,2015$.

22 Subik K, Lee JF, Baxter L, Strzepek T, Costello D, Crowley P, Xing L, Hung MC, Bonfiglio T, Hicks DG and Tang P: The expression patterns of ER, PR, HER2, CK5/6, EGFR, Ki-67 and AR by immunohistochemical analysis in breast cancer cell lines. Breast Cancer 4: 35-41, 2010.

23 Zhou JH, Kang N, Cui LX and He W: Selection of culture media for the mass production of gamma delta T-cells used in adoptive immunotherapy. Zhongguo Yi Xue Ke Xue Yuan Xue Bao 33: 644-648, 2011.

24 Kim EK, Ahn YO, Kim S, Kim TM, Keam B and Heo DS: Ex vivo activation and expansion of natural killer cells from patients with advanced cancer with feeder cells from healthy volunteers. Cytotherapy 15: 231-241.e231, 2013.

25 Kolanowski ST, Sritharan L, Lissenberg-Thunnissen SN, Van Schijndel GM, Van Ham SM and ten Brinke A: Comparison of media and serum supplementation for generation of monophosphoryl lipid A/interferon-gamma-matured type I dendritic cells for immunotherapy. Cytotherapy 16: 826-834, 2014.

26 Peek R, Bastings L, Westphal JR, Massuger LF, Braat DD and Beerendonk CC: A preliminary study on a new model system to evaluate tumour-detection and tumour-purging protocols in ovarian cortex tissue intended for fertility preservation. Hum Reprod 30: 870-876, 2015.

27 Schroder CP, Timmer-Bosscha H, Wijchman JG, de Leij LF, Hollema H, Heineman MJ and de Vries EG: An in vitro model for purging of tumour cells from ovarian tissue. Hum Reprod 19: 1069-1075, 2004.

$28 \mathrm{Hu}$ G, Pen W and Wang M: TRIM14 promotes breast cancer cell proliferation by inhibiting apoptosis. Oncol Res, 2018. doi: 10.3727/096504018X15214994641786. [Epub ahead of print]

$29 \mathrm{Wu}$ L, Li Y, Li J and Ma D: MicroRNA-664 targets insulin receptor substrate 1 to suppress cell proliferation and invasion in breast cancer. Oncol Res, 2018. doi: 10.3727/096504 018X15193500663936. [Epub ahead of print]

30 Bockstaele L, Tsepelidis S, Dechene J, Englert $\mathrm{Y}$ and Demeestere I: Safety of ovarian tissue autotransplantation for cancer patients. Obstet Gynecol Int 2012: 1-6, 2012.

31 Kyono K, Doshida M, Toya M, Sato Y, Akahira J and Sasano H: Potential indications for ovarian autotransplantation based on the analysis of 5,571 autopsy findings of females under the age of 40 in Japan. Fertil Steril 93: 2429-2430, 2010.

Received October 1, 2018

Revised October 16, 2018

Accepted October 24, 2018 